\title{
Numerical Stability of the Halley-Iteration for the Solution of a System of Nonlinear Equations
}

\author{
By Annie A. M. Cuyt*
}

\begin{abstract}
Let $F: \mathbf{R}^{q} \rightarrow \mathbf{R}^{q}$ and $x^{*}$ a simple root in $\mathbf{R}^{q}$ of the system of nonlinear equations $F(x)=0$.

Abstract Padé approximants (APA) and abstract Rational approximants (ARA) for the operator $F$ have been introduced in [2] and [3]. The adjective "abstract" refers to the use of abstract polynomials [5] for the construction of the rational operators.

The APA and ARA have been used for the solution of a system of nonlinear equations in [4]. Of particular interest was the following third order iterative procedure:

$$
x_{i+1}=x_{i}+\frac{a_{i}^{2}}{a_{i}+\frac{1}{2} F_{i}^{\prime-1} F_{i}^{\prime \prime} a_{i}^{2}},
$$

with $F_{i}^{\prime}$ the 1st Fréchet-derivative of $F$ in $x_{i}, a_{i}=-F_{i}^{\prime-1} F_{i}$ the Newton-correction where $F_{i}=F\left(x_{i}\right), F_{i}^{\prime \prime}$ the 2nd Fréchet-derivative of $F$ in $x_{i}$ where $F_{i}^{\prime \prime} a_{i}^{2}$ is the bilinear operator $F_{i}^{\prime \prime}$ evaluated in $\left(a_{i}, a_{i}\right)$, and componentwise multiplication and division in $\mathbf{R}^{q}$. For $q=1$ this technique is known as the Halley-iteration [6, p. 91]. In this paper the numerical stability [7] of the Halley-iteration for the case $q>1$ is investigated and illustrated by a numerical example.
\end{abstract}

1. Numerical Stability of Iterations. We consider the numerical solution of the equation

$$
F(x)=0
$$

with $F: \mathbf{R}^{q} \rightarrow \mathbf{R}^{q}: x \rightarrow F(x)$, abstract analytic in 0 [5]. Assume that (1) has a simple root $x^{*}$.

We briefly repeat the definition of condition-number given by Woźniakowski [7]. The condition-number should measure the sensitivity of the solution (output) with respect to changes in the data (input). We assume that $F$ depends parametrically on a vector $d \in \mathbf{R}^{p}$, called data vector

$$
F(x)=F(x ; d),
$$

and instead of the exact value $F(x ; d)$ we only have the computed value $\mathrm{fl}(F(x ; d))$ in $t$ digit floating-point binary arithmetic. At best we can expect that $\mathrm{fl}(F(x ; d))$ is the exact value of a slightly perturbed operator at slightly perturbed data

$$
\mathrm{fl}(F(x ; d))=(I+\Delta F) F(x+\Delta x ; d+\Delta d),
$$

where $I$ is the $q \times q$ unit-matrix and

$$
\begin{gathered}
\|\Delta x\| \leqslant C_{1} \rho\|x\|, \quad\|\Delta d\| \leqslant C_{2} \rho\|d\|, \\
\|\Delta F\| \leqslant C_{3} \rho \quad(\Delta F \text { a } q \times q \text { matrix }),
\end{gathered}
$$

Received May 27, 1981.

1980 Mathematics Subject Classification. Primary 65G05.

* Aspirant of the Belgian National Fund for Scientific Research (NFWO). 
for constants $C_{1}, C_{2}, C_{3}$ (only depending on the dimensions of the problem) and with $\rho=2^{-t}$ the relative computer precision [8]. By introducing the Landau-symbol $O$, we could also write

$$
\Delta x=O(\rho), \quad \Delta d=O(\rho), \Delta F=O(\rho),
$$

where the constants in the Landau-notation depend on $x, d$ and the dimensions. We will always, for a given $F$, define the data vector so that (2) holds and so that the condition number (see Definition 1.1) is minimized. Let $\mathrm{fl}(d)$ denote the $t$ digit binary representation of the vector $d$ in floating-point arithmetic

$$
\|\mathrm{fl}(d)-d\| \leqslant C \rho\|d\|, \quad \text { i.e. } \mathrm{fl}(d)-d=O(\rho) .
$$

Since $d$ is represented by $\mathrm{fl}(d)$, we solve in fact $F(x ; \mathrm{fl}(d))=0$ instead of $F(x)=0$, independent of the method used to solve (1). Let $F_{x}^{\prime}$ and $F_{d}^{\prime}$ denote the partial Fréchet-derivatives of $F$, respectively with respect to $x$ and $d$.

Now $F(x ; \mathrm{fl}(d))=0$ has a root $\widetilde{x}^{*}$ in the neighborhood of $x^{*}$ and $\widetilde{x}^{*}-x^{*}=$ $O(\rho)$ if $t$ is sufficiently large; thus,

$$
\begin{aligned}
\widetilde{x^{*}-x^{*}=} & -F_{x}^{\prime}\left(x^{*} ; d\right)^{-1} F_{d}^{\prime}\left(x^{*} ; d\right)(\mathrm{fl}(d)-d) \\
& + \text { higher order terms in } \widetilde{x^{*}}-x^{*} \text { and } \mathrm{fl}(d)-d \\
= & -F_{x}^{\prime}\left(x^{*} ; d\right)^{-1} F_{d}^{\prime}\left(x^{*} ; d\right)(\mathrm{fl}(d)-d)+O\left(\rho^{2}\right),
\end{aligned}
$$

where the constant in the Landau-notation depends on $x^{*}, d$ and $F$. For $x^{*} \neq 0:\left\|\widetilde{x^{*}}-x^{*}\right\| /\left\|x^{*}\right\| \leqslant\left\|F_{x}^{\prime}\left(x^{*} ; d\right)^{-1} F_{d}^{\prime}\left(x^{*} ; d\right)\right\| C \rho\|d\| /\left\|x^{*}\right\|+O\left(\rho^{2}\right)$.

Definition 1.1. Cond $(F ; d)=\left\|F_{x}^{\prime}\left(x^{*} ; d\right)^{-1} F_{d}^{\prime}\left(x^{*} ; d\right)\right\| \cdot\|d\| /\left\|x^{*}\right\|$ is called the condition number of $F$ with respect to the data vector $d$.

A problem is ill-conditioned if $\operatorname{cond}(F ; d) \gg 1$.

Let us now suppose that $F(x ; d)=0$ is solved by an iterative procedure $\Phi\left(x_{i}, F\right)$, where $\Phi$ can use several $F_{i}^{(j)}$, the $j$ th Fréchet-derivative of $F$ at $x_{i}$ (if $j=1$ or 2 , a single or double prime is used instead of the superscript $j$ ). If $\left\{x_{i}\right\}$ is the sequence of successive approximations of $x^{*}$, we can at best expect $x_{i}$ to be the representation of a computed value for $\widetilde{x^{*}}$,

$$
\left\|x_{i}-\widetilde{x^{*}}\right\| \leqslant K \rho\left\|\widetilde{x^{*}}\right\|
$$

So

$$
\begin{aligned}
\left\|x_{i}-x^{*}\right\| & \leqslant\left\|x_{i}-\widetilde{x^{*}}\right\|+\left\|\widetilde{x^{*}}-x^{*}\right\| \leqslant K \rho\left\|\widetilde{x^{*}}\right\|+C \rho \operatorname{cond}(F ; d) \cdot\left\|x^{*}\right\|+O\left(\rho^{2}\right) \\
& \leqslant K \rho\left(\left\|\widetilde{x^{*}}-x^{*}\right\|+\left\|x^{*}\right\|\right)+C \rho \operatorname{cond}(F ; d) \cdot\left\|x^{*}\right\|+O\left(\rho^{2}\right) \\
& \leqslant[K \rho+C \rho \operatorname{cond}(F ; d)] \cdot\left\|x^{*}\right\|+O\left(\rho^{2}\right) .
\end{aligned}
$$

Definition 1.2. An iteration $\Phi$ is called numerically stable if

$$
\lim _{i \rightarrow \infty}\left\|x_{i}-x^{*}\right\| \leqslant \rho \cdot\left\|x^{*}\right\| \cdot(C \operatorname{cond}(F ; d)+K)+O\left(\rho^{2}\right)
$$

where the constants $C$ and $K$ depend on $x^{*}, d$ and $F$.

In practice we often want to find an approximation $x_{i}$ such that $\left\|x_{i}-x^{*}\right\| \leqslant \varepsilon$. $\left\|x^{*}\right\|$. This is possible if the problem is sufficiently well-conditioned, i.e., $\rho \operatorname{cond}(F ; d)=O(\varepsilon)$. In floating-point arithmetic we have

$$
x_{i+1}=\Phi\left(x_{i}, F\right)+\xi_{i}, \quad \text { where } \xi_{i}=\mathrm{fl}\left(\Phi\left(x_{i}, F\right)\right)-\Phi\left(x_{i}, F\right) .
$$


THEOREM 1.1. A convergent iterative procedure $\Phi\left(x_{i}, F\right)$, i.e.

$$
\lim _{i \rightarrow \infty}\left\|\Phi\left(x_{i}, F\right)-x^{*}\right\|=0,
$$

is numerically stable if $\lim _{i \rightarrow \infty}\left\|\xi_{i}\right\| \leqslant \rho\left\|x^{*}\right\|(C \operatorname{cond}(F ; d)+K)+O\left(\rho^{2}\right)$.

Proof. We simply verify the definition.

$$
\begin{aligned}
\lim _{i \rightarrow \infty}\left\|x_{i}-x^{*}\right\| & \leqslant \lim _{i \rightarrow \infty}\left[\left\|\Phi\left(x_{i-1}, F\right)-x^{*}\right\|+\left\|\xi_{i-1}\right\|\right] \\
& =\lim _{i \rightarrow \infty}\left\|\xi_{i-1}\right\| \leqslant \rho\left\|x^{*}\right\|(C \operatorname{cond}(F ; d)+K)+O\left(\rho^{2}\right) .
\end{aligned}
$$

\section{Abstract Padé Approximants (APA) and Abstract Rational Approximants (ARA)} for the Solution of a System of Nonlinear Equations. Let $x_{i}$ be the $i$ th approximant of the root $x^{*}$ in the iterative process, $y_{i}=F\left(x_{i}\right)$ and the Newton-correction $a_{i}=-F_{i}^{\prime-1} F_{i}$. Using the Inversion Theorem [1, p. 381] we can see that

$$
x^{*}=x_{i}+a_{i}-\frac{1}{2} F_{i}^{\prime-1} F_{i}^{\prime \prime} a_{i}^{2}+O\left(a_{i}^{3}\right),
$$

where $F_{i}^{\prime \prime} a_{i}^{2}$ is the bilinear operator $F_{i}^{\prime \prime}$ evaluated on $\left(a_{i}, a_{i}\right)$. The Newton-iteration results from approximating the series in (3) by its first two terms, i.e., the $(1,0)$-APA [2].

In [7] Woźniakowski proves numerical stability of the Newton-iteration under a natural assumption on the computed evaluation of $F$.

THEOREM 2.1. If

(a) $\mathrm{fl}\left(F\left(x_{i} ; d\right)\right)=\left(I+\Delta F_{i}\right) F\left(x_{i}+\Delta x_{i} ; d+\Delta d_{i}\right)=F\left(x_{i}\right)+\delta F_{i}$, with

$$
\delta F_{i}=\Delta F_{i} F\left(x_{i}\right)+F_{x}^{\prime}\left(x_{i}\right) \Delta x_{i}+F_{d}^{\prime}\left(x_{i}\right) \Delta d_{i}+O\left(\rho^{2}\right) \text {, }
$$

(b) $\mathrm{fl}\left(F^{\prime}\left(x_{i} ; d\right)\right)=F^{\prime}\left(x_{i}\right)+\delta F_{i}^{\prime}$, with $\delta F_{i}^{\prime}=O(\rho)$,

(c) the computed correction $\mathrm{fl}\left(a_{i}\right)$ is the exact solution of a perturbed linear system

$$
\left(F^{\prime}\left(x_{i}\right)+\delta F_{i}^{\prime}+E_{i}\right) \mathrm{fl}\left(a_{i}\right)=-F\left(x_{i}\right)-\delta F_{i} \text { with } E_{i}=O(\rho)
$$

then the Newton-iteration is numerically stable.

Proof. In [7].

Another way to approximate $x^{*}$ is to use the $(1,1)$-ARA [2] for the power series (3), i.e.

$$
x_{i+1}=x_{i}+\frac{a_{i}^{2}}{a_{i}+\frac{1}{2} F_{i}^{\prime-1} F_{i}^{\prime \prime} a_{i}^{2}},
$$

where multiplication and division of the vectors in $\mathbf{R}^{q}$ in the numerator and denominator of (4) are componentwise. For $q=1$ the iteration (4) is the wellknown Halley-iteration. We will also use the name Halley-iteration for the case $q \geqslant 1$. We will now prove numerical stability of this iteration under assumptions similar to the assumptions for the Newton-iteration. We will also assume that the divisions in (4) are such that

$$
\left(\frac{1}{a_{i}+\frac{1}{2} F_{i}^{\prime-1} F_{i}^{\prime \prime} a_{i}^{2}}\right)^{j} O\left(\left\|a_{i}\right\|^{j-k} \rho^{k+l}\right)=O\left(\rho^{l}\right) .
$$


Condition (5) takes care of the fact that the denominator of the correction-term in (4) does not become too small in comparison with $O\left(\left\|a_{i}\right\|^{j-k} \rho^{k}\right)$.

The assumption of (5) is a natural generalization of the following relations:

$$
\begin{aligned}
& \text { for } q=1, \lim _{i \rightarrow \infty} \frac{a_{i}}{a_{i}+\frac{1}{2} F_{i}^{\prime-1} F_{i}^{\prime \prime} a_{i}^{2}}=1, \\
& \text { and so } \exists L \in \mathrm{N} \supset \quad \forall i \geqslant L:\left|\frac{a_{i}}{a_{i}+\frac{1}{2} F_{i}^{\prime-1} F_{i}^{\prime \prime} a_{i}^{2}}\right|<1+D \\
& \text { (case } j=1, k=0, l=0 \text { ) with } D \in \mathbf{R}_{0}^{+} \text {, }
\end{aligned}
$$

in a convergent process (4): $\lim _{i \rightarrow \infty}\left\|x^{*}-x_{i}\right\|=0$, and thus

$$
\begin{aligned}
& \lim _{i \rightarrow \infty} a_{i}=0 \text {, i.e. } \exists M \in \mathbf{N} \supset \forall i \geqslant M: a_{i}=O(\rho) \text {, } \\
& \text { and so } \forall i \geqslant M: a_{i}^{2}=O\left(\left\|a_{i}\right\| \rho\right) \text {; also } \\
& \lim _{i \rightarrow \infty} \frac{a_{i}^{2}}{a_{i}+\frac{1}{2} F_{i}^{\prime-1} F_{i}^{\prime \prime} a_{i}^{2}}=0 \text {, i.e. } \\
& \exists N \in \mathbf{N} \supset \forall i \geqslant N: \frac{a_{i}^{2}}{a_{i}+\frac{1}{2} F_{i}^{\prime-1} F_{i}^{\prime \prime} a_{i}^{2}}=O(\rho), \\
& \text { and so } \forall i \geqslant \max (N, M) \text { : } \frac{a_{i}^{2}}{a_{i}+\frac{1}{2} F_{i}^{\prime-1} F_{i}^{\prime \prime} a_{i}^{2}} \\
& =\frac{1}{a_{i}+\frac{1}{2} F_{i}^{\prime-1} F_{i}^{\prime \prime} a_{i}^{2}} O\left(\left\|a_{i}\right\| \rho\right) \\
& =O(\rho) \\
& \text { (case } j=1, k=0, l=1) \text {. }
\end{aligned}
$$

THEOREM 2.2. If

(a) $\mathrm{fl}\left(F\left(x_{i} ; d\right)\right)=\left(I+\Delta F_{i}\right) F\left(x_{i}+\Delta x_{i} ; d+\Delta d_{i}\right)=F\left(x_{i}\right)+\delta F_{i}$ with

$$
\delta F_{i}=\Delta F_{i} F\left(x_{i}\right)+F_{x}^{\prime}\left(x_{i}\right) \Delta x_{i}+F_{d}^{\prime}\left(x_{i}\right) \Delta d_{i}+O\left(\rho^{2}\right) \text {, }
$$

(b) $\mathrm{fl}\left(F^{\prime}\left(x_{i} ; d\right)\right)=F^{\prime}\left(x_{i}\right)+\delta F_{i}^{\prime}$ with $\delta F_{i}^{\prime}=O(\rho)$,

(c) $\mathrm{fl}\left(F^{\prime \prime}\left(x_{i} ; d\right)\right)=F^{\prime \prime}\left(x_{i}\right)+\delta F_{i}^{\prime \prime}$ with $\delta F_{i}^{\prime \prime}=O(\rho)$,

(d) the computed correction $\mathrm{fl}\left(a_{i}\right)$ is the exact solution of a perturbed linear system

$$
\left(F^{\prime}\left(x_{i}\right)+\delta F_{i}^{\prime}+E_{i, 1}\right) \mathrm{fl}\left(a_{i}\right)=-F\left(x_{i}\right)-\delta F_{i} \text { with } E_{i, 1}=O(\rho),
$$

(e) analogously,

$$
\begin{aligned}
\left(F^{\prime}\left(x_{i}\right)+\delta F_{i}^{\prime}+E_{i, 2}\right) \mathrm{fl}\left(b_{i}\right)= & \left(F^{\prime \prime}\left(x_{i}\right)+\delta F_{i}^{\prime \prime}\right) \mathrm{fl}\left(a_{i}\right)^{2} \\
& \text { with } E_{i, 2}=O(\rho) \text { and } b_{i}=F_{i}^{\prime-1} F_{i}^{\prime \prime} a_{i}^{2},
\end{aligned}
$$

and (5) holds, then the iteration (4) is numerically stable.

$$
\begin{array}{r}
\text { Proof. Let } F^{\prime}\left(x_{i}\right)+\delta F_{i}^{\prime}+E_{i, 1}=F^{\prime}\left(x_{i}\right)\left(I+H_{i, 1}\right) \text {, where } \\
H_{i, 1}=F^{\prime}\left(x_{i}^{\prime}\right)^{-1}\left\{\delta F_{i}^{\prime}+E_{i, 1}\right\}=O(\rho)
\end{array}
$$

because of (b) and (d). So for small $\rho$,

$$
\left(I+H_{i, 1}\right)^{-1}=I-H_{i, 1}+O\left(\rho^{2}\right) .
$$


Thus

$$
\mathrm{fl}\left(a_{i}\right)=\left(I-H_{i, 1}\right) F_{i}^{\prime-1}\left(-F_{i}-\delta F_{i}\right)
$$

Analogously

$$
\mathrm{fl}\left(b_{i}\right)=\left(I-H_{i, 2}\right) F_{i}^{\prime-1}\left(F_{i}^{\prime \prime}+\delta F_{i}^{\prime \prime}\right) \mathrm{fl}\left(a_{i}\right)^{2} \text { with } H_{i, 2}=O(\rho) .
$$

Now

$$
\begin{aligned}
\left(F_{i}^{\prime \prime}+\right. & \left.\delta F_{i}^{\prime \prime}\right) \mathrm{fl}\left(a_{i}\right)^{2}=\left(F_{i}^{\prime \prime}+\delta F_{i}^{\prime \prime}\right)\left[\left(I-H_{i, 1}\right) F_{i}^{\prime-1}\left(-F_{i}-\delta F_{i}\right)\right]^{2} \\
& =\left(F_{i}^{\prime \prime}+\delta F_{i}^{\prime \prime}\right) a_{i}^{2}+2\left(F_{i}^{\prime \prime}+\delta F_{i}^{\prime \prime}\right)\left(F_{i}^{\prime-1} F_{i}, F_{i}^{\prime-1} \delta F_{i}-H_{i, 1} F_{i}^{\prime-1} F_{i}\right)+O\left(\rho^{2}\right) \\
& =\left(F_{i}^{\prime \prime}+\delta F_{i}^{\prime \prime}\right) a_{i}^{2}-2 F_{i}^{\prime \prime}\left(a_{i}, F_{i}^{\prime-1} \delta F_{i}-H_{i, 1} F_{i}^{\prime-1} F_{i}\right)+O\left(\rho^{2}\right) .
\end{aligned}
$$

Thus

$$
\begin{aligned}
\mathrm{fl}\left(b_{i}\right)= & F_{i}^{\prime-1}\left(F_{i}^{\prime \prime}+\delta F_{i}^{\prime \prime}\right) a_{i}^{2}-2 F_{i}^{\prime-1} F_{i}^{\prime \prime}\left(a_{i},{F_{i}^{\prime}}^{-1} \delta F_{i}-H_{i, 1} F_{i}^{\prime-1} F_{i}\right) \\
& -H_{i, 2} F_{i}^{\prime-1} F_{i}^{\prime \prime} a_{i}^{2}+O\left(\rho^{2}\right) .
\end{aligned}
$$

A computed approximation $x_{i+1}$ satisfies

$$
x_{i+1}=\left(I+\delta I_{i, 1}\right)\left(x_{i}+\left(I+\delta I_{i, 2}\right) \frac{\mathrm{fl}\left(a_{i}\right)^{2}}{\mathrm{fl}\left(a_{i}\right)+\frac{1}{2} \mathrm{fl}\left(b_{i}\right)}\right),
$$

where $\delta I_{i, 1}$ and $\delta I_{i, 2}$ are diagonal matrices and $\delta I_{i, 1}=O(\rho)$ and $\delta I_{i, 2}=O(\rho)$. So

$$
x_{i+1}=\left(I+\delta I_{i, 1}\right)\left(x_{i}+\left(I+\delta I_{i, 2}\right) \frac{a_{i}^{2}-2 a_{i} \cdot\left(F_{i}^{\prime-1} \delta F_{i}+H_{i, 1} a_{i}\right)+O\left(\rho^{2}\right)}{a_{i}+\frac{1}{2} b_{i}-\delta a_{i}+O\left(\rho^{2}\right)}\right],
$$

where

$$
\begin{aligned}
\delta a_{i}= & F_{i}^{\prime-1} \delta F_{i}+H_{i, 1} a_{i}-\frac{1}{2}{F_{i}^{\prime-1}}^{\prime} \delta F_{i}^{\prime \prime} a_{i}^{2} \\
& +\frac{1}{2} H_{i, 2} F_{i}^{\prime-1} F_{i}^{\prime \prime} a_{i}^{2}+{F_{i}^{\prime}}^{-1} F_{i}^{\prime \prime}\left(a_{i},{F_{i}^{\prime}}^{-1} \delta F_{i}-H_{i, 1} F_{i}^{\prime-1} F_{i}\right) .
\end{aligned}
$$

Using (6), we find

$$
\mathrm{fl}\left(a_{i}\right)-a_{i}+H_{i, 1} a_{i}-H_{i, 1} F_{i}^{\prime-1} \delta F_{i}=-F_{i}^{\prime-1} \delta F_{i},
$$

and thus, for positive constants $D_{1}$ and $D_{2}$,

$$
\left\|F_{i}^{\prime-1} \delta F_{i}\right\| \leqslant D_{2} \rho\left\|a_{i}\right\| \quad \text { since }\left\|\mathrm{fl}\left(a_{i}\right)-a_{i}\right\|<D_{1} \rho\left\|a_{i}\right\|
$$

and

$$
\left\|F_{i}^{\prime-1}\right\| \cdot\left\|F_{i}\right\| \leqslant\left\|F_{i}^{\prime-1}\right\| \cdot\left\|F_{i}^{\prime}\right\| \cdot\left\|a_{i}\right\|
$$

Thus

$$
x_{i+1}=\left(I+\delta I_{i, 1}\right)\left(x_{i}+\frac{a_{i}^{2}-2 a_{i}\left(F_{i}^{\prime-1} \delta F_{i}+H_{i, 1} a_{i}\right)+\delta I_{i, 2} a_{i}^{2}+O\left(\rho^{2}\left\|a_{i}\right\|^{2}\right)}{a_{i}+\frac{1}{2} b_{i}-\delta a_{i}+O\left(\rho^{2}\right)}\right),
$$

where $\delta I_{i, 2} a_{i}^{2}$ is the linear operator $\delta I_{i, 2}$ evaluated in $a_{i}^{2}$ (componentwise square of the vector $\left.a_{i}\right)$. So

$$
x_{i+1}=\left(I+\delta I_{i, 1}\right)\left[x_{i}+\frac{a_{i}^{2}-2 a_{i}\left(F_{i}^{\prime-1} \delta F_{i}+H_{i, 1} a_{i}\right)+\delta I_{i, 2} a_{i}^{2}+O\left(\rho^{2}\left\|a_{i}\right\|^{2}\right)}{a_{i}+\frac{1}{2} b_{i}} \cdot c_{i}\right),
$$


with

$$
c_{i}=1+\frac{1}{a_{i}+\frac{1}{2} b_{i}}\left(\delta a_{i}+O\left(\rho^{2}\right)\right)+\left(\frac{1}{a_{i}+\frac{1}{2} b_{i}}\right)^{2} O\left(\left\|a_{i}\right\|^{2-k} \rho^{k+2}, k=0,1,2\right)
$$

since $\delta a_{i}=O\left(\rho\left\|a_{i}\right\|\right)$; in $c_{i}$ we have used the notation 1 for the unit vector $(1, \ldots, 1)$.

Using (5), we conclude

$$
\left(\frac{1}{a_{i}+\frac{1}{2} b_{i}}\right)^{2} O\left(\left\|a_{i}\right\|^{2-k} \rho^{k+2}, k=0,1,2\right)=O\left(\rho^{2}\right) .
$$

For $\xi_{i}=x_{i+1}-\Phi\left(x_{i}, F\right)$, we have

$$
\begin{aligned}
\xi_{i}= & \delta I_{i, 1} x_{i}+\frac{a_{i}^{2}}{a_{i}+\frac{1}{2} b_{i}}\left(c_{i}-1\right) \\
& +\frac{-2 a_{i}\left(F_{i}^{\prime-1} \delta F_{i}+H_{i, 1} a_{i}\right)+\delta I_{i, 2} a_{i}^{2}+O\left(\rho^{2}\left\|a_{i}\right\|^{2}\right)}{a_{i}+\frac{1}{2} b_{i}} \cdot c_{i} \\
& +\delta I_{i, 1} \frac{a_{i}^{2}}{a_{i}+\frac{1}{2} b_{i}} \cdot c_{i}+O\left(\rho^{2}\right) .
\end{aligned}
$$

So

$$
\begin{aligned}
\xi_{i}= & \delta I_{i, 1} x_{i}+\left(\frac{1}{a_{i}+\frac{1}{2} b_{i}}\right)^{2} O\left(\rho\left\|a_{i}\right\|^{3}, \rho^{2}\left\|a_{i}\right\|^{2}\right)+\frac{1}{a_{i}+\frac{1}{2} b_{i}} O\left(\rho^{2}\left\|a_{i}\right\|^{2}\right) \\
& +\frac{1}{a_{i}+\frac{1}{2} b_{i}}\left(-2 a_{i} F_{i}^{\prime-1} \delta F_{i}+O\left(\rho\left\|a_{i}\right\|^{2}, \rho^{2}\left\|a_{i}\right\|^{2}\right)\right) \cdot(1+O(\rho)) \\
& +O\left(\rho^{2}\right) .
\end{aligned}
$$

Thus

$$
\left\|\xi_{i}\right\| \leqslant k_{1} \rho\left\|x_{i}\right\|+k_{2} \rho\left\|a_{i}\right\|+\left\|\frac{-2 a_{i}}{a_{i}+\frac{1}{2} b_{i}} F_{i}^{\prime-1} \delta F_{i}\right\|+O\left(\rho^{2}\right),
$$

and since

$$
\begin{aligned}
\frac{-2 a_{i}}{a_{i}+\frac{1}{2} b_{i}} F_{i}^{\prime-1} \delta F_{i}= & \frac{-2 a_{i}}{a_{i}+\frac{1}{2} b_{i}} F_{i}^{\prime-1}\left(\Delta F_{i} F\left(x_{i}\right)+F_{i}^{\prime} \Delta x_{i}+F_{d}^{\prime} \Delta d_{i}+O\left(\rho^{2}\right)\right) \\
= & \frac{1}{a_{i}+\frac{1}{2} b_{i}} O\left(\rho\left\|a_{i}\right\|\right) F\left(x_{i}\right)-\frac{2 a_{i}}{a_{i}+\frac{1}{2} b_{i}} \Delta x_{i} \\
& -\frac{2 a_{i}}{a_{i}+\frac{1}{2} b_{i}} F_{i}^{\prime-1} F_{d}^{\prime} \Delta d_{i}+\frac{1}{a_{i}+\frac{1}{2} b_{i}} O\left(\rho^{2}\left\|a_{i}\right\|\right)
\end{aligned}
$$

we find that

$$
\lim _{i \rightarrow \infty}\left\|\xi_{i}\right\| \leqslant \rho\left\|x^{*}\right\|(K+C \operatorname{cond}(F ; d))+O\left(\rho^{2}\right)
$$

for $\lim _{i \rightarrow \infty} a_{i}=0=\lim _{i \rightarrow \infty} F\left(x_{i}\right)$ in a convergent process and $a_{i} \Delta x_{i}=O\left(\rho\left\|a_{i}\right\|\right)$ and $a_{i} F_{i}^{\prime-1} F_{d}^{\prime} \Delta d_{i}=O\left(\rho\left\|a_{i}\right\|\right)$. 
3. Numerical Example. Consider the following operator:

$$
F: \mathbf{R}^{2} \rightarrow \mathbf{R}^{2}:(x, y) \rightarrow\left(\begin{array}{l}
e^{-x+y}-d_{1} \\
e^{-x-y}-d_{2}
\end{array}\right) \text { with } d_{1}>0 \text { and } d_{2}>0 .
$$

The operator $F$ has a simple root $x^{*}=\left(-\frac{1}{2} \ln \left(d_{1} d_{2}\right), \frac{1}{2} \ln \left(d_{1} / d_{2}\right)\right)$. Clearly

$$
d=\left(d_{1}, d_{2}\right)
$$

is the data vector. Now

$$
\mathrm{fl}(F(x, y ; d))=\left(\begin{array}{l}
{\left[\left(1+\varepsilon_{1}\right) e^{\left(-x-\Delta^{\prime} x+y+\Delta^{\prime} y\right)\left(1+\theta_{1}\right)}-\left(d_{1}+\Delta_{1}^{\prime} d\right)\right]\left(1+\kappa_{1}\right)} \\
{\left[\left(1+\varepsilon_{2}\right) e^{\left(-x-\Delta^{\prime} x-y-\Delta^{\prime} y\right)\left(1+\theta_{2}\right)}-\left(d_{2}+\Delta_{2}^{\prime} d\right)\right]\left(1+\kappa_{2}\right)}
\end{array}\right),
$$

where $\mathrm{fl}(x)=x+\Delta^{\prime} x, \mathrm{fl}(y)=y+\Delta^{\prime} y, \mathrm{fl}\left(d_{1}\right)=d_{1}+\Delta_{1}^{\prime} d, \mathrm{fl}\left(d_{2}\right)=d_{2}+\Delta_{2}^{\prime} d, \theta_{1}$ is caused by $-\mathrm{fl}(x)+\mathrm{fl}(y), \theta_{2}$ is caused by $-\mathrm{fl}(x)-\mathrm{fl}(y), \varepsilon_{i}$ are caused by the exponential evaluations $(i=1,2), \kappa_{i}$ are caused by the subtraction of $\operatorname{fl}\left(d_{i}\right)$ $(i=1,2)$.

One can rewrite $\mathrm{fl}(F(x, y ; d))=(I+\Delta F) F(x+\Delta x, y+\Delta y ; d+\Delta d)$ with

$$
\begin{gathered}
\Delta x=x \theta_{1}+\Delta^{\prime} x\left(1+\theta_{1}\right), \quad \Delta y=y \theta_{1}+\Delta^{\prime} y\left(1+\theta_{1}\right), \quad \Delta d=\left(\Delta_{1} d, \Delta_{2} d\right), \\
\Delta_{1} d=\frac{\Delta_{1}^{\prime} d-\varepsilon_{1} d_{1}}{1+\varepsilon_{1}}, \\
\Delta F=\left(\begin{array}{cc}
\left(1+\varepsilon_{1}\right)\left(1+\kappa_{1}\right)-1 & \left(1+\varepsilon_{2}\right)\left(1+\kappa_{2}\right) e^{\left(x+\Delta^{\prime} x+y+\Delta^{\prime} y\right)\left(\theta_{1}-\theta_{2}\right)}-1 \\
0 & \left(1+\varepsilon_{2}\right.
\end{array}\right) .
\end{gathered}
$$

The inverse of the Jacobian matrix in the root $x^{*}$ is

$$
\frac{1}{2\left(d_{1} \cdot d_{2}\right)}\left(\begin{array}{cc}
-d_{2} & -d_{1} \\
d_{2} & -d_{1}
\end{array}\right) \text { and } F_{d}^{\prime}=\left(\begin{array}{cc}
-1 & 0 \\
0 & -1
\end{array}\right)
$$

The condition number of $F$ with respect to the data vector $d$ is

$$
\left\|F_{x}^{\prime}\left(x^{*} ; d\right)^{-1}\right\| \cdot \frac{\left\|\left(d_{1}, d_{2}\right)\right\|}{\left\|x^{*}\right\|} \text {. }
$$

Using the Schur-norm $\|A\|=\sqrt{\sum_{i, j} a_{i j}^{2}}$ of a matrix $A=\left(a_{i j}\right)$ and the $l_{2}$-norm $\|a\|=\sqrt{\sum_{i} a_{i}^{2}}$ of a vector $a=\left(a_{i}\right)$, the condition number is

$$
\frac{d_{1}^{2}+d_{2}^{2}}{\sqrt{2} d_{1} \cdot d_{2} \cdot\left\|x^{*}\right\|}
$$

Putting $d_{1}=d=d_{2}$, the root $x^{*}=(-\ln d, 0)$ and the condition number is $\sqrt{2} /|\ln d|$. The problem is extremely well-conditioned if $\operatorname{cond}(F ; d) \leqslant 1$, i.e.,

$$
\left.d \in]-\infty, e^{-\sqrt{2}}\right] \cup\left[e^{\sqrt{2}},+\infty[\text {. }\right.
$$

The problem is very ill-conditioned if $d=e^{\varepsilon}$ with $\varepsilon$ very small. We will now check some of the conditions of Theorem 2.2. We already $\operatorname{know} \mathrm{fl}(F(x, y ; d))=$ $(I+\Delta F) F(x+\Delta x, y+\Delta y ; d+\Delta d)$.

Now

$$
\mathrm{fl}\left(F^{\prime}(x, y ; d)\right)=\mathrm{fl}\left(\begin{array}{cc}
-e^{-x+y} & e^{-x+y} \\
-e^{-x-y} & -e^{-x-y}
\end{array}\right)
$$


where

$$
\begin{aligned}
\mathrm{fl}\left(e^{-x+y}\right)= & \left(1+\varepsilon_{1}\right) e^{\left(-x-\Delta^{\prime} x+y+\Delta^{\prime} y\right)\left(1+\theta_{1}\right)}=\left(1+\varepsilon_{1}\right) e^{-x+y} e^{-\Delta x+\Delta y} \\
= & e^{-x+y}\left[1+\varepsilon_{1}+\left(1+\varepsilon_{1}\right)\left(e^{-\Delta x+\Delta y}-1\right)\right] \\
\mathrm{fl}\left(e^{-x-y}\right)= & \left(1+\varepsilon_{2}\right) e^{\left(-x-\Delta^{\prime} x-y-\Delta^{\prime} y\right)\left(1+\theta_{2}\right)} \\
= & \left(1+\varepsilon_{2}\right) e^{-x-y} e^{-\Delta x-\Delta y} e^{\left(x+\Delta^{\prime} x+y+\Delta^{\prime} y\right)\left(\theta_{1}-\theta_{2}\right)} \\
= & e^{-x-y}\left[1+\varepsilon_{2}+\left(1+\varepsilon_{2}\right)\left(e^{-\Delta x-\Delta y} e^{\left(x+\Delta^{\prime} x+y+\Delta^{\prime} y\right)\left(\theta_{1}-\theta_{2}\right)}-1\right)\right] .
\end{aligned}
$$

\begin{tabular}{|c|c|c|c|c|}
\hline k & $x_{6}$ & $Y_{6}$ & $\ell$ & $\operatorname{cond}\left(F ; e^{10^{-K}}\right)$ \\
\hline 0 & $-0.1000000000000000(01)$ & $0.3597855161523896(-18)$ & 16 & $\sqrt{2}$ \\
\hline 1 & $-0.1000000000000000(00)$ & $-0.2376055789464463(-17)$ & 16 & $10 \sqrt{2}$ \\
\hline 2 & $-0.1000000000000001(-01)$ & $-0.6397150159689099(-17)$ & 15 & $10^{2} \sqrt{2}$ \\
\hline 3 & $-0.0999999999999997(-02)$ & $0.5077502606368951(-17)$ & 15 & $10^{3} \sqrt{2}$ \\
\hline 4 & $-0.0999999999999844(-03)$ & $0.3913464269882279(-17)$ & 13 & $10^{4} \sqrt{2}$ \\
\hline 5 & $-0.0999999999997470(-04)$ & $-0.3905797959965137(-17)$ & 12 & $10^{5} \sqrt{2}$ \\
\hline 6 & $-0.0999999999986935(-05)$ & $0.5633677343553680(-17)$ & 11 & $10^{6} \sqrt{2}$ \\
\hline 7 & $-0.1000000000174599(-06)$ & $-0.1058449777227516(-16)$ & 10 & $10^{7} \sqrt{2}$ \\
\hline 8 & $-0.1000000000015281(-07)$ & $0.4124494865312562(-17)$ & 11 & $10^{8} \sqrt{2}$ \\
\hline 9 & $-0.1000000007452433(-08)$ & $-0.2449359520991520(-17)$ & 9 & $10^{9} \sqrt{2}$ \\
\hline 10 & $-0.0999999914314586(-09)$ & $0.4265833288825851(-17)$ & 8 & $10^{10} \sqrt{2}$ \\
\hline 11 & $-0.1000000261210709(-10)$ & $-0.6446772724219823(-17)$ & 7 & $10^{11} \sqrt{2}$ \\
\hline 12 & $-0.0999980430668081(-11)$ & $0.3302303528672576(-17)$ & 5 & $10^{12} \sqrt{2}$ \\
\hline 13 & $-0.0999761308551817(-12)$ & $0.1322187990417560(-16)$ & 4 & $10^{13} \sqrt{2}$ \\
\hline 14 & $-0.1000372750236664(-13)$ & $-0.1182870095748150(-16)$ & 4 & $10^{14} \sqrt{2}$ \\
\hline 15 & $-0.0963108239652912(-14)$ & $0.1398012990192197(-17)$ & 2 & $10^{15} \sqrt{2}$ \\
\hline 16 & $-0.0868560967896870(-15)$ & $0.3349523961106902(-17)$ & 1 & $10^{16} \sqrt{2}$ \\
\hline
\end{tabular}

So $\mathrm{fl}\left(F^{\prime}(x, y ; d)\right)=F^{\prime}(x, y ; d)+\delta F^{\prime}(x, y ; d)$ with

$$
\begin{aligned}
& \delta F^{\prime}(x, y ; d) \\
&=\left(\begin{array}{cc}
\varepsilon_{1}+\left(1+\varepsilon_{1}\right)\left(e^{-\Delta x+\Delta y}-1\right) & 0 \\
0 & \varepsilon_{2}+\left(1+\varepsilon_{2}\right)\left(e^{-\Delta x-\Delta y} e^{\left(x+\Delta^{\prime} x+y+\Delta^{\prime} y\right)\left(\theta_{1}-\theta_{2}\right)}-1\right)
\end{array}\right) \\
& \cdot F^{\prime}(x, y ; d)=O(\rho) .
\end{aligned}
$$

We can write down an analogous formula for $F^{\prime \prime}(x, y ; d)$.

We remark that the algorithm even behaves considerably well for a condition number of the order of $10^{3}$ or $10^{4}$.

The two linear systems of equations are well-conditioned since the condition number of the linear systems in $x^{*}=\lim _{i \rightarrow \infty} x_{i}$ is

$$
\left\|F_{x}^{\prime}\left(x^{*} ; d\right)^{-1}\right\| \cdot\left\|F_{x}^{\prime}\left(x^{*} ; d\right)\right\|=2 .
$$

One can prove that the use of Gaussian elimination with row pivoting for this example satisfies the conditions (d) and (e) of Theorem 2.2. So we can expect to get a reasonable approximation of the solution of $F(x, y ; d)=0$ using the numerically stable iterative method (4); the numerical results illustrate this. Let us at the same time follow the loss of significant digits in the root $x^{*}$ as the problem becomes worse-conditioned. The calculations are performed in double precision $(t=56)$ on the PDP 11/45 of the University of Antwerp. We will solve the nonlinear system 
$F(x, y ; d)=0$ for $d=e^{10^{-k}}, k=0, \ldots, 16$. The root $x^{*}=\left(-10^{-k}, 0\right)$. For each $d$ we give the 6th iteration-step $\left(x_{6}, y_{6}\right)$ in the procedure (4) starting from $\left(x_{0}, y_{0}\right)=$ $(2,2)$, the number $l$ of significant digits in $x_{6}$, and the condition number $\operatorname{cond}\left(F ; e^{10^{-k}}\right)$. It is also important to know that the iterative procedure stops at the 6th iteration-step, except for $k=7,13$, and 14 where, respectively, $l=11,5$, and 3 in the last iteration-step $\left(x_{7}, y_{7}\right)$. We have used the stop-criterion

$$
\max \left(\left|x_{i+1}-x_{i}\right|,\left|y_{i+1}-y_{i}\right|\right) \leqslant 10^{-15} \max \left(\left|x_{i+1}\right|,\left|y_{i+1}\right|\right) \text {. }
$$

Department of Mathematics

University of Antwerp, U.I.A.

Universiteitsplein 1

B-2610 Wilrijk, Belgium

1. R. G. BARTLE, The Elements of Real Analysis, Wiley, New York, 1976.

2. ANNIE A. M. CUYT, Abstract Padé Approximants in Operator Theory, Lecture Notes in Math., Vol. 765, Springer-Verlag, Berlin and New York, 1979, pp. 61-87.

3. ANNIE A. M. CUYT, "On the properties of Abstract Rational (1-point) Approximants," J. Operator Theory, v. 5, 1981. (To appear.)

4. A. CUYt \& P. VAN Der CRuYsSen, Abstract Padé Approximants for the Solution of a System of Nonlinear Equations, Report 80-17, University of Antwerp, 1980.

5. Lous B. RALl, Computational Solution of Nonlinear Operator Equations, Wiley, New York, 1969; reprinted by Krieger, Huntington, New York, 1979.

6. J. F. Traub, Iterative Methods for the Solution of Equations, Prentice-Hall, Englewood Cliffs, N. J., 1964.

7. H. Woźnıakowskı, "Numerical stability for solving nonlinear equations," Numer. Math., v. 27, 1977, pp. 373-390.

8. D. Young, A Survey of Numerical Mathematics. I, Addison-Wesley, Reading, Mass., 1972. 